\title{
Anterior urethra sparing cystoprostatectomy for bladder cancer: a 10-year, single center experience
}

\author{
Nozomi Hayakawa ${ }^{1,2^{*}}$, Nobuyuki Kikuno ${ }^{1,2}$, Hiroki Ishihara ${ }^{1,2}$, Osamu Ryoji ${ }^{1,2}$ and Kazunari Tanabe ${ }^{2}$
}

\begin{abstract}
Purpose: Decision making regarding the urethra before and after radical cystectomy due to urothelial carcinoma has always been controversial. To determine whether anterior urethra sparing cystoprostatectomy for bladder cancer is an oncologically-safe procedure, we evaluated the long-term oncologic clinical outcome.

Patients and methods: A total of 51 male patients with cTa-4NO-2MO bladder cancer were treated with anterior urethra sparing cystoprostatectomy and simultaneous urinary diversion between 2000 and 2013, and underwent follow up for 4 months or more. We assessed differences in the perioperative outcomes, oncologic outcomes and recurrence rates according to the urinary diversion.

Results: The median patient age and follow-up period were 66 years and 35 months, respectively. The 5- and 10-year recurrence free survival (RFS) rates in ileal conduit (IC) group vs. orthotopic neobladder reconstruction (NB) group were 45.0 and $20.3 \%$ vs. 39.3 and 19.6\%, respectively. Likewise, the 5 - and 10-year disease specific survival (DSS) were 52.7 and $32.1 \%$ vs. 39.3 and 29.5\%, respectively. Multivariate analysis revealed two independent prognostic factors for RFS and DSS, including age at surgery and lymph node status. Local recurrence in the remnant anterior urethra occurred in only 1 patient (2.0\%) at 57 months after surgery.
\end{abstract}

Conclusions: Our long-term data show that anterior urethra sparing cystoprostatectomy is an oncologically-safe procedure regardless of the type of urinary diversion in a subset of carefully selected patients with bladder cancer without evidence of urothelial carcinoma in the urethra/bladder neck and urethral surgical margin.

Keywords: Bladder cancer, Cystectomy, Urethra, Urinary diversion

\section{Background}

Radical cystoprostatectomy is the standard treatment in men with muscle-invasive bladder cancer (MIBC) or refractory high risk non-MIBC (Gakis et al. 2013; Stenzl et al. 2011). This includes removal of the bladder, prostate, seminal vesicles and vasa deferentia (Gakis et al. 2013). The overall incidence of urethral recurrence (UR) in contemporary cystectomy patients is $4-6 \%$. In patients undergoing orthotopic bladder replacement, the incidence is lower (Huguet 2013; Huguet et al. 2008; Huguet 2012; Stein et al. 2005; Boorjian et al. 2011). The most

\footnotetext{
*Correspondence: nozomih0331@gmail.com

1 Department of Urology, Saiseikai Kawaguchi General Hospital,

Nishikawaguchi, Kawaguchi, Saitama, Japan

Full list of author information is available at the end of the article
}

consistent risk factor associated with the occurrence of UR is the involvement with urothelial prostate tumor, and especially, prostatic stromal involvement (Stein et al. 2005; Nieder et al. 2004; Hardeman and Soloway 1990).

Perineal urethrectomy is indicated with cystectomy in male patients in the presence of urethral involvement by cancer or if there is a risk of urethral reinvolvement, such as a positive prostatic urethral margin, prostatic urethral superficial urothelial carcinoma (UC) or prostatic stromal invasion (Cho et al. 2009). It has been also reported to be a more complicating procedure as a result of the additional perineal wound, longer operation duration and delayed ambulation (Elshal et al. 2011). Therefore, it remains a controversial matter whether simultaneous prophylactic urethrectomy or urinary diversion with

\section{垈 Springer}

(C) 2015 Hayakawa et al. This article is distributed under the terms of the Creative Commons Attribution 4.0 International License (http://creativecommons.org/licenses/by/4.0/), which permits unrestricted use, distribution, and reproduction in any medium, provided you give appropriate credit to the original author(s) and the source, provide a link to the Creative Commons license, and indicate if changes were made. 
radical cystoprostatectomy should be performed even for cases in which the principal risk factors for UR have been identified preoperatively. Moreover, there are few reports of remnant urethral recurrence in male patients undergoing ileal conduit with no prophylactic urethrectomy.

We present our experience in evaluating whether anterior urethra sparing cystoprostatectomy for bladder cancer influences into the incidence of remnant urethral recurrence and subsequent oncologic clinical outcome, based upon differences of simultaneous urinary diversion.

\section{Results and discussion}

Clinical presentation, diagnosis, and pathological findings Ultimately, a study population of 51 males with cTa4N0-2M0 bladder cancer undergoing anterior urethra sparing cystoprostatectomy and simultaneous urinary diversion was eligible for analysis. The patient characteristics are summarized in Additional file 1: Table S1. The median age of the patients was 66 years (range 48-84 years). Of the 56 consecutive patients, 5 patients were excluded for the following reasons: 2 patients had no urinary diversion caused by chronic renal failure requiring maintenance hemodialysis among their comorbid disorders, 2 patients had undergone percutaneous ureterostomy as urinary diversion and bone metastasis, and the another one patient was diagnosed as having squamous cell carcinoma post-pathologically. IC and NB were selected as the approach to urinary diversion in 25 (49.0\%) and 26 (51.0\%) patients, respectively. The median follow up time was 35 months (range 4-143 months). Upon review of the final cystoprostatectomy specimen, the presence of carcinoma in situ (CIS), non-organ confined disease (pT3 or greater), and node-positive disease were evident in 10 (19.6\%), 19 (37.3\%), and 9 (17.6\%) patients, respectively. A total of four patients (7.9\%) demonstrated prostatic urethral involvement. While one patient had pathological prostatic ductal disease, three exhibited stromal involvement. There were no differences in the presence of CIS, pathological stage, UC grade, and prostate involvement when comparing patients undergoing IC vs. NB urinary diversion.

\section{Perioperative outcomes and complications}

The median operation time was significantly shorter for the IC group (546 min) compared with the NB group (594 $\min )(P=0.027)$, However, there were no statistical differences in the median blood loss or the number of hospitalization days between these subgroups (1,500 vs. $1,700 \mathrm{ml}$ and 47 vs. 41 days in the IC group vs. NB group, respectively) (Additional file 2: Table S2). The surgical margins were tumor free for all cases.

While there was no perioperative mortality, a total of $19(37.3 \%)$ patients experienced perioperative complications. Both ileus and pyelonephritis occurred as the most frequent complication in $4(7.8 \%)$ patients each. There was no significant impact on the complication rate and type between these subgroups (Additional file 3: Table S3).

\section{Oncologic outcomes and recurrence rates according to the} site in patients with postoperative recurrence

Five- and 10-year RFS by Kaplan-Meier estimation in IC group vs. NB group were 45.0 and $20.3 \%$ vs. 39.3 and $19.6 \%$, respectively ( $\mathrm{p}>0.05$ ) (Fig. 1). Similarly, the 5- and 10-year DSS were 52.7 and $32.1 \%$ vs. 39.3 and $29.5 \%$, respectively ( $\mathrm{p}>0.05$ ) (Fig. 2). Multivariate analysis revealed three independent prognostic factors for RFS, including age at surgery $(\mathrm{p}=0.03)$, maximum pathological tumor stage $(\mathrm{p}=0.01)$, lymph node status $(\mathrm{p}=0.0001)$ and two independent prognostic factors for DSS, including age at surgery $(\mathrm{p}=0.006)$, lymph node status $(\mathrm{p}=0.03$ ) (Additional file 4: Table S4). None of the other factors tested proved to be of prognostic
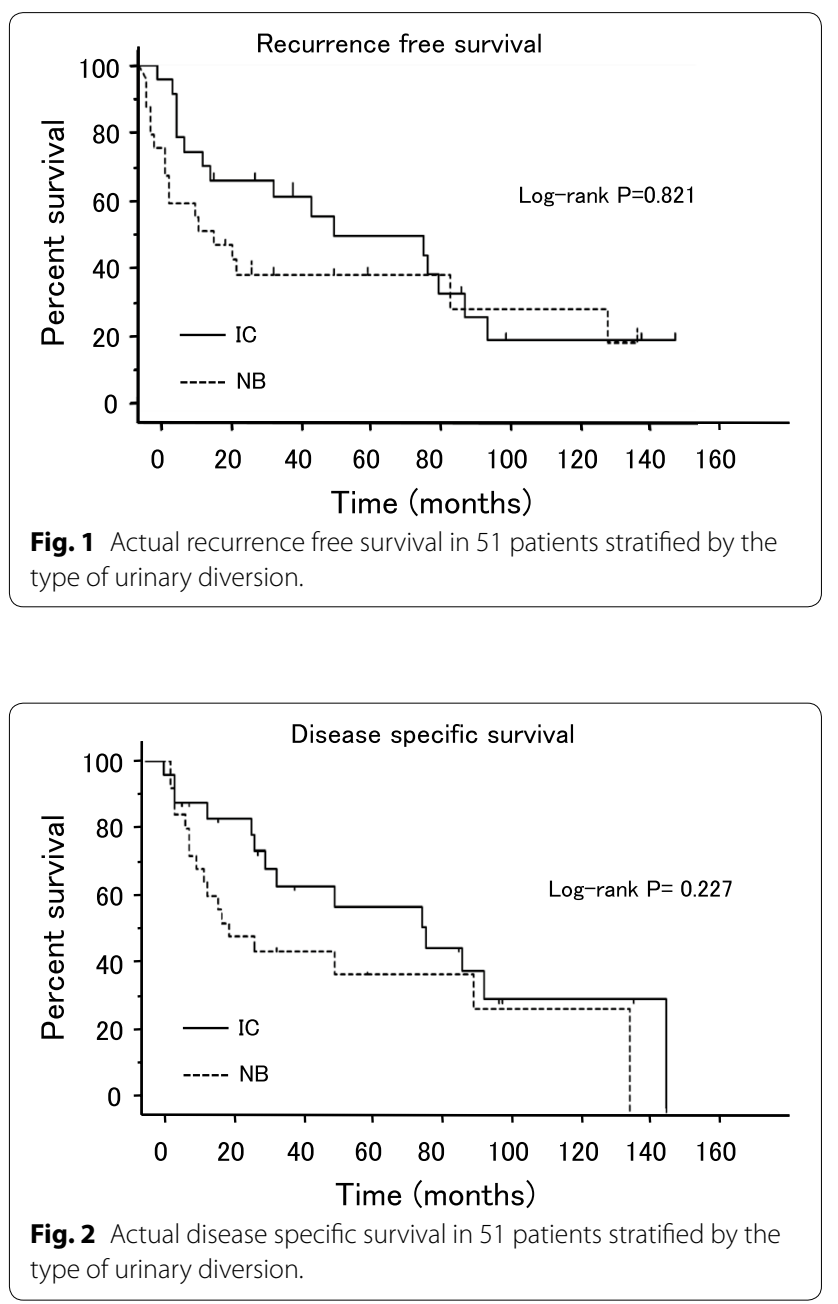
significance. Patients with lymph node-positive disease or who were over 60 years old demonstrated significantly worse survival and higher recurrence rates than those with no lymph node involvement or who were under 60 years of age $(p=0.013$ and 0.001 or $p=0.001$ and 0.001 , respectively). Recurrence developed during follow-up in 20 of the 51 patients (39.2\%), including distant recurrence of abdominopelvic lymph node, lung, bone, liver and local recurrence of muscle, rectum, ureter and remnant urethra (Additional file 5: Table S5). A total of 9 patients (17.6\%), comprised of 6 in the IC group and 3 in the NB group presented with distant, as well as local recurrences. Remnant urethral recurrence was identified in only one patient in the NB group (2.0\%) at 57 postoperative months, although other recurrence sites were more frequently confirmed during the same follow-up period. There were no significant differences in the recurrence rates according to the site in patients with postoperative recurrence between each subgroup.

Decision making regarding the urethra before and after radical cystectomy due to urothelial carcinoma has always been controversial (Huguet 2011). The incidence of urethral recurrence of UC after radical cystectomy has been estimated to be as high as 10\% (Freeman et al. 1994). Significant attention has been directed toward determining appropriate patient selection for urethral preservation and orthotopic neobladder reconstruction. It is generally accepted that orthotopic diversion should not be performed if there is a high risk of tumor recurrence in the retained urethra. While the pathological features considered as potentially high risk have evolved during the last decade, with some of these factors surgeons continue to consider prophylactic urethrectomy, thereby precluding orthotopic diversion (Hassan et al. 2004). These characteristics include prostatic urethral involvement in men, bladder neck and/or vaginal wall involvement in women and CIS (Freeman et al. 1996). By contrast, there have been few reports in which intraoperative frozen section biopsies of the urethral margin have been used systematically and with sufficient follow-up; although most authors currently agree that this approach would determine whether orthotopic diversion or urethrectomy should be performed (Huguet 2011). Kassouf et al. (2008) reported that a positive preoperative transurethral resection prostatic urethral biopsy did not correlate with final margin and should not exclude patients from consideration for orthotopic urinary diversion in their study of 245 radical cystectomy specimens for UC. There are also a few reports of remnant urethral recurrence in male patients who had undergone the ileal conduit procedure without prophylactic urethrectomy. Based on these reports, we hypothesized that simultaneous prophylactic urethrectomy with radical cystoprostatectomy might not always be necessary, regardless of the type of urinary diversion, if the urethral margin alone was negative on frozen section. In our series, surgical margins were confirmed to be tumor free for all cases. Therefore, prophylactic urethrectomy was not performed and the types of urinary diversion were divided into IC and NB groups, according to the complete assessment of several factors, including patient age, patient desire, preoperative complications, past history of digestive disease, intraoperative findings, such as the length of the mesentery or the presence of some adhesion. As shown in Additional file 1: Table S1, there were no significant differences in the patient background among those patients undergoing IC vs. NB urinary diversion. As a result, the histopathological findings in our retrospective study revealed that 4 patients (7.9\%) exhibited prostatic involvement even if the negative urethral surgical margin was confirmed intraoperatively. However, remnant urethral recurrence was identified in only one patient $(2.0 \%)$ who interestingly exhibited no prostatic involvement.

In recent years, surgeons have begun to report case series of minimally invasive radical cystectomy for the treatment of bladder cancer, including laparoscopic radical cystectomy (LRC) and robot-assisted radical cystectomy (RARC), demonstrating the surgical feasibility of these procedure with the potential of reduced blood loss and more rapid return of bowel function and hospital discharge (Nix et al. 2010; Challacombe et al. 2011). LRC and RARC are increasingly performed; although open radical cystectomy (ORC) remains the standard approach for bladder cancer (Yuh et al. 2015; Stenzl et al. 2011; Snow-Lisy et al. 2014; Bochner et al. 2014; Linder et al. 2014). Furthermore, Tyritzis et al. (2013) reported that outcomes after RARC with totally intracorporeal neobladder diversion appeared satisfactory and consistent with a contemporary open series. Despite these excellent experiences and observations, high levels of clinical evidence with respect to the significance of urethral management, such as LRC or RARC with simultaneous prophylactic urethrectomy, are lacking. Perineal urethrectomy has been reported to be a complicating procedure as a consequence of the extra perineal wound, longer operation duration and delayed ambulation, although radical cysto-urethrectomy is recommended for every patient with bladder cancer who has a urethral tumor or a risk of urethral reinvolvement (Elshal et al. 2011). Under this recent trend, minimally invasive surgical techniques have achieved increasing support and the decision making process regarding the urethra before and after cystectomy due to bladder cancer has become an even more difficult problem.

In our study, patients with lymph node-positive disease or those who were over 60 years old demonstrated 
significantly worse survival and higher recurrence rates than those with no lymph node involvement or those who were younger than 60 years. However, there were no statistically significant differences in perioperative outcomes, except for the median operation time, oncologic outcomes and recurrence rates based upon the urinary diversion approach. Remnant urethral recurrence was identified in only one patient $(2.0 \%)$. These results are similar to the previous reports (Taylor et al. 2010; Hautmann et al. 2006). Our data indicate that anterior urethra sparing cystoprostatectomy for bladder cancer did not influence the incidence of remnant urethral recurrence and oncologic clinical outcome based upon different approaches to simultaneous urinary diversion. We believe that our study could be very useful in consideration of the significance of simultaneous prophylactic urethrectomy with radical cystectomy, regardless of LRC, RARC, or ORC.

Our study is limited by the retrospective, non-randomized design and by the small number of patients. A randomized clinical trial focused on the definitive urethral management before and after minimally invasive radical cystectomy will be necessary in the future.

\section{Conclusions}

Our long-term data indicate that anterior urethra sparing cystoprostatectomy is an oncologically-safe procedure, regardless of the type of urinary diversion, in a subset of carefully selected patients with bladder cancer without evidence of urothelial carcinoma in the urethra/bladder neck or the urethral surgical margin.

\section{Methods}

\section{Study population}

We retrospectively reviewed the medical records of 56 consecutive male patients who underwent an anterior urethra sparing cystoprostatectomy for primary bladder cancer at our institute between December 2000 and September 2013, who were included in a computerized database containing comprehensive clinical and pathological data. All female patients were excluded. Patients were excluded from analysis when they did not have pathological evidence of urothelial carcinoma or did not undergo IC or NB. The final pathological analysis was performed using routine step sectioning, which was the protocol during this period. The primary bladder lesion was classified using the TNM classification (Edge et al. 2010).

\section{Treatment and follow-up}

Indication for radical cystectomy were muscle invasive bladder cancer (MIBC) or high-risk non MIBC (NMIBC) including multifocal T1 tumors, T1 tumors located in some difficult sites for complete resection, residual T1 after re-resection, T1 with concomitant CIS, and BCGrefractory tumors. This study cohort had undergone anterior urethra sparing cystoprostatectomy and urinary diversion. Several forms of urinary diversion were selected, as follows: IC, NB and ureterocutaneostomy. Patient follow-up was relatively uniform and included urinary cytology, abdomen-pelvis computed tomography, whole-body bone scan, and chest X-ray. These tests were performed at 3-month intervals for the initial 2 years, at 6-month intervals for the subsequent 3 years, and annually thereafter. Recurrence was confirmed by pathology findings and/or based on imaging findings in all patients classified as exhibiting tumor recurrence. The diagnosis of urethral recurrence was confirmed pathologically in the resected transurethral tissue samples.

\section{Study methods}

Clinical information was obtained by a retrospective review of all patient medical records. Preoperative tumor stage was determined according to the UICC. We investigated $\mathrm{T}$ stage, $\mathrm{N}$ stage, $\mathrm{M}$ stage, tumor grade, concomitant CIS, and urethral surgical margin status. To rule out macroscopic tumor formation in the bladder neck, posterior or anterior urethra, all patients underwent preoperative urethrocystoscopy. Negative urethral surgical margins were confirmed by pathological diagnosis during surgery. The clinical and pathological characteristics of these patients are described in Additional file 1: Table S1.

\section{Outcomes}

We assessed the perioperative outcomes (operation time, blood loss, the number of hospitalization days, perioperative complications), oncologic outcomes, including RFS and DSS, and recurrence rates according to the different approaches to simultaneous urinary diversion. RFS and DSS were defined as the time from anterior urethra sparing cystoprostatectomy with simultaneous urinary diversion until recurrence and the date of death from bladder cancer or last follow up, respectively. Patients were monitored until death or loss to follow-up; the median values are reported for age at the time of operation and the duration of follow-up.

\section{Statistical analysis}

Results were analyzed using descriptive statistical analysis. Survival data were analyzed using Kaplan-Meier survival estimates. The log-rank test was used to compare RFS and DSS in the patient subgroups. Cox proportional hazard regression models were used to evaluate independent prognostic factors associated with RFS and DSS in this patient cohort. Stat mate III for Windows was used for the statistical analyses and two-sided p-values $<0.05$ were considered significant. 


\section{Additional files}

Additional file 1: Table S1. Demographic and pathological characteristics of 51 male patients undergoing anterior urethra sparing cystoprostatectomy and simultaneous urinary diversion for bladder UC.

Additional file 2: Table S2. Perioperative outcomes.

Additional file 3: Table S3. Perioperative complications.

Additional file 4: Table S4. Multivariate analysis of factors associated with RFS and DSS.

Additional file 5 Table S5. Recurrence rates according to the site in patients with postoperative recurrence.

\section{Abbreviations}

MIBC: muscle-invasive bladder cancer; UR: urethral recurrence; UC: urothelial carcinoma; CIS: carcinoma in situ; IC: ileal conduit; NB: orthotopic neobladder reconstruction; LRC: Iaparoscopic radical cystectomy; RARC: robot-assisted radical cystectomy; ORC: open radical cystectomy; UICC: Unio Internationalis Contra Cancrum; RFS: recurrence free survival; DSS: disease specific survival.

\section{Author's contributions}

$\mathrm{NH}$ conceived of the study, collected the data, performed the statistical analysis, and drafted the manuscript. NK helped to perform the statistical analysis and drafted the manuscript. HI collected the data. OR participated in the design of the study. KT participated in the design of the study. All authors read and approved the final manuscript.

\section{Author details}

${ }^{1}$ Department of Urology, Saiseikai Kawaguchi General Hospital, Nishikawaguchi, Kawaguchi, Saitama, Japan. ${ }^{2}$ Department of Urology, Tokyo Women's Medical University, Shinjyuku-ku, Tokyo, Japan.

\section{Acknowledgements}

I would like to thank all the patients that made it possible to complete this study.

\section{Compliance with ethical guidelines}

\section{Competing interests}

The authors declare that they have no competing interests.

Received: 9 February 2015 Accepted: 29 July 2015

Published online: 08 August 2015

\section{References}

Bochner BH, Dalbagni G, Sjoberg DD, Silberstein J, Keren Paz GE, Donat SM et al (2014) Comparing open radical cystectomy and robot-assisted laparoscopic radical cystectomy: a randomized clinical trial. Eur Urol. doi:10.1016/j.eururo.2014.11.043

Boorjian SA, Kim SP, Weight CJ, Cheville JC, Thapa P, Frank I (2011) Risk factors and outcomes of urethral recurrence following radical cystectomy. Eur Urol 60:1266-1272

Challacombe BJ, Bochner BH, Dasgupta P et al (2011) The role of laparoscopic and robotic cystectomy in the management of muscle-invasive bladder cancer with special emphasis on cancer control and complications. Eur Urol 60:767-775

Cho KS, Seo JW, Park SJ, Lee YH, Choi YD, Cho NH et al (2009) The risk factor for urethral recurrence after radical cystectomy in patients with transitional cell carcinoma of the bladder. Urol Int 82:306-311
Edge SB, Compton CC, The American Joint Committee on Cancer (2010) The 7th edition of the AJCC cancer staging manual and the future of TNM. Ann Surg Oncol 17:1471-1474

Elshal AM, Barakat TS, Mosbah A, Abdel-Latif M, Abol-Enein H (2011) Complications of radical cysto-urethrectomy using modified Clavien grading system: prepubic versus perineal urethrectomy. BJU Int 108:1297-1300

Freeman JA, Esrig D, Stein JP, Skinner DG (1994) Management of the patient with bladder cancer. Urethral recurrence. Urol Clin North Am 21:645-651

Freeman JA, Tarter TA, Esrig D et al (1996) Urethral recurrence in patients with orthotopic ileal neobladders. J Urol 156:1615-1619

Gakis G, Efstathiou J, Lerner SP et al (2013) ICUD-EAU International Consultation on Bladder Cancer 2012: Radical cystectomy and bladder preservation for muscle-invasive urothelial carcinoma of the bladder. Eur Urol 63:45-57

Hardeman SW, Soloway MS (1990) Urethral recurrence following radical cystectomy. J Urol 144:666-669

Hassan JM, Cookson MS, Smith JA Jr, Chang SS (2004) Urethral recurrence in patients following orthotopic urinary diversion. J Urol 172:1338-1341

Hautmann RE, Gschwend JE, de Petriconi RC, Kron M, Volkmer BG (2006) Cystectomy for transitional cell carcinoma of the bladder: results of a surgery only series in the neobladder era. J Urol 176:486-492

Huguet J (2011) Management of the male urethra before and after cystectomy: from the prophylactic urethrectomy to the intraoperative frozen section biopsy of the urethral margin. Actas Urol Esp 35:552-558

Huguet J (2012) Diagnosis and treatment of urethral recurrence after radical cystectomy in the male. Actas Urol Esp 36:42-47

Huguet J (2013) Follow-up after radical cystectomy based on patterns of tumor recurrence and its risk factors. Actas Urol Esp 37:376-382

Huguet J, Monllau V, Sabaté S et al (2008) Diagnosis, risk factors, and outcome of urethral recurrences following radical cystectomy for bladder cancer in 729 male patients. Eur Urol 53:785-793

KassoufW, Spiess PE, Brown GA, Liu P, Grossman HB, Dinney CP et al (2008) Prostatic urethral biopsy has limited usefulness in counseling patients regarding final urethral margin status during orthotopic neobladder reconstruction. J Urol 180:164-167

Linder BJ, Boorjian SA, Hudolin TJ, Cheville JC, Thapa P, Tarrell RF et al (2014) Late recurrence after radical cystectomy: patterns, risk factors and outcomes. Urol 191:1256-1261

Nieder AM, Sved PD, Gomez P, Kim SS, Manoharan M, Soloway MS (2004) Urethral recurrence after cystoprostatectomy: implications for urinary diversion and monitoring. Urology 64:950-954

Nix J, Smith A, Kurpad R, Nielsen ME, Wallen EM, Pruthi RS (2010) Prospective randomized controlled trial of robotic versus open radical cystectomy for bladder cancer: perioperative and pathologic results. Eur Urol 57:196-201

Snow-Lisy DC, Campbell SC, Gill IS, Hernandez AV, Fergany A, Kaouk J et al (2014) Robotic and laparoscopic radical cystectomy for bladder cancer: long-term oncologic outcomes. Eur Urol 65:193-200

Stein JP, Clark P, Miranda G, Cai J, Groshen S, Skinner DG (2005) Urethral tumor recurrence following cystectomy and urinary diversion: clinical and pathological characteristics in 768 male patients. J Urol 173:1163-1168

Stenzl A, Cowan NC, De Santis M, Kuczyk MA, Merseburger AS, Ribal MJ, et al (2011) Treatment of muscle-invasive and metastatic bladder cancer: update of the EAU guidelines. Eur Urol 59:1009-1018

Taylor JM, Spiess PE, Kassouf W, Munsell MF, Kamat AM, Dinney CP et al (2010) Management of urethral recurrence after orthotopic urinary diversion. BJU Int 106:56-61

Tyritzis SI, Hosseini A, Collins J, Nyberg T, Jonsson MN, Laurin O et al (2013) Oncologic, functional, and complications outcomes of robot-assisted radical cystectomy with totally intracorporeal neobladder diversion. Eur Urol 64:734-741

Yuh B, Wilson T, Bochner B, Chan K, Palou J, Stenzl A et al (2015) Systematic review and cumulative analysis of oncologic and functional outcomes after robot-assisted radical cystectomy. Eur Urol 67(3):402-422. doi:10.1016/j.eururo.2014.12.008 